\title{
Recent heavy ion results from the ATLAS experiment
}

\author{
Mariusz Przybycien ${ }^{1, \star}$ (on behalf of the ATLAS Collaboration) \\ ${ }^{1}$ AGH University of Science and Technology, Faculty of Physics and Applied Computer Science, \\ Al. Mickiewicza 30, 30-059 Cracow, Poland
}

\begin{abstract}
The ATLAS experiment at the LHC has undertaken a broad physics program to probe and characterize the hot nuclear matter created in relativistic lead-lead collisions. This overview presents recent results on production of electroweak bosons, charmonia, charged-particles, jets, and bulk particle collectivity from $\mathrm{Pb}+\mathrm{Pb}$ and $p+\mathrm{Pb}$ collisions at $2.76 \mathrm{TeV}$ and $5.02 \mathrm{TeV}$, respectively, as well as on electromagnetic processes in ultraperipheral $\mathrm{Pb}+\mathrm{Pb}$ collisions at $5.02 \mathrm{TeV}$.
\end{abstract}

\section{Introduction}

The Quark-Gluon Plasma (QGP) was first observed in experiments at RHIC [1-4] and since then it has been intensively studied in relativistic heavy-ion (HI) collisions. Significantly higher collision energies available at the LHC compared to RHIC, result in increased volume, lifetime and temperature of QGP. We are particularly interested in understanding of the collision geometry and centrality as well as in how the free nucleon parton distribution functions are modified in the nuclear environment. One can study properties of QGP using hard probes of different scales (electroweak bosons, jets, heavy quarks) or measuring parameters describing collective behaviour of the medium. Hard probes are produced early in the $\mathrm{HI}$ collision, in a process which cross section is not changed by presence of strongly interacting medium, i.e. it can by calculated using perturbative QCD. Passing through the medium hard probes interact weakly or strongly with it providing information on its properties. Due to their large electric charge heavy-ions are also strong sources of high energy photons. Thus, in ultra-peripheral collisions, in which strong interactions are suppressed, one can study two-photon interactions. A review of HI results from LHC experiments based on Run 1 data can be found in [5]. In these Proceedings an overview of the recent HI results from the ATLAS experiment [6] is presented based on $\mathrm{Pb}+\mathrm{Pb}$ collision data at $\sqrt{s_{\mathrm{NN}}}=2.76 \mathrm{TeV}$ and $p+\mathrm{Pb}$ collision data at $\sqrt{s_{\mathrm{NN}}}=5.02 \mathrm{TeV}$.

Yields of hard processes in HI collisions are expected to scale with the number of binary nucleonnucleon collisions, $N_{\text {coll }}$, which depends on the centrality of the collision. In the central collisions, when the overlap of nuclei is large, one expects a high number of $N_{\text {coll }}$ and a high number of nucleons participating in the collision, $N_{\text {part }}$. On the other hand, in more peripheral collisions, when nuclei overlap only partly, both $N_{\text {coll }}$ and $N_{\text {part }}$ are expected to be smaller.

A proxy to the QGP volume produced in a collision is the collision centrality. In ATLAS centrality of events is determined by the sum of transverse energy deposited in the forward calorimeter (FCal) covering the pseudorapidity range $3.2<|\eta|<4$.8. In case of $\mathrm{Pb}+\mathrm{Pb}$ collisions the sum on both sides

\footnotetext{
^e-mail: Mariusz.Przybycien@agh.edu.pl
} 
and in case of $p+\mathrm{Pb}$ collisions the sum on the $\mathrm{Pb}$-going side only are taken into account $[7,8]$. The centrality intervals are expressed in percentiles of the total inelastic non-Coulomb $\mathrm{Pb}+\mathrm{Pb}$ or $p+\mathrm{Pb}$ cross sections, respectively. For the correlation between a centrality interval and the average number of participants, $\left\langle N_{\text {part }}\right\rangle$, the Glauber model is used for $\mathrm{Pb}+\mathrm{Pb}$ collisions and both the Glauber and Glauber-Gribov colour fluctuation [9] models for $p+\mathrm{Pb}$ collisions.

A variable often used to quantify the modification of rates of hard processes in a nuclear environment with respect to $p p$ collisions is the nuclear modification factor:

$$
R_{\mathrm{AA}}=\frac{1}{\left\langle T_{\mathrm{AA}}\right\rangle} \frac{1 / N_{\mathrm{evt}} \mathrm{d}^{2} N_{\mathrm{Pb}+\mathrm{Pb}} / \mathrm{d} \eta \mathrm{d} p_{\mathrm{T}}}{\mathrm{d}^{2} \sigma_{p p} / \mathrm{d} \eta \mathrm{d} p_{\mathrm{T}}}
$$

where in the numerator is the yield of a given process per minimum-bias (MB) event in HI collisions, possibly in a given centrality interval, and in the denominator the $p p$ cross section for the same process. The $T_{\mathrm{AA}}$ is the mean nuclear thickness function and is a measure of the nuclear overlap i.e. of the number of nucleons which can participate in the hard scattering processes.

\section{Electroweak bosons and charmonia}

Electroweak (EW) bosons being colourless do not interact strongly with QGP, and their production rates are therefore expected to be directly sensitive to the overall thickness of the colliding nuclear matter. The rates are also expected to be sensitive to modifications of the partonic structure of nucleons bound in a nucleus, which are implemented as nuclear modifications to the parton distribution functions (PDFs) in the proton. These effects include nuclear shadowing (depletion of the parton densities at low Bjorken $x$ ), antishadowing (an enhancement at moderate $x$ ), the EMC effect and Fermi motion [10].

ATLAS has studied production of $W$ and $Z$ bosons in both $\mathrm{Pb}+\mathrm{Pb}[11,12]$ and in $p+\mathrm{Pb}$ collisions $[13,14]$. In $\mathrm{Pb}+\mathrm{Pb}$ collisions $W$ bosons were measured in both electron and muon $(\ell=e, \mu)$ decay channels in the fiducial phase space: $p_{\mathrm{T}}^{\ell}>25 \mathrm{GeV}, p_{\mathrm{T}}^{\text {miss }}>25 \mathrm{GeV}, m_{\mathrm{T}}>40 \mathrm{GeV}$ and extrapolated to $\left|\eta_{\ell}\right|<2.5$. $W$ boson production yields per binary collision for each charge separately as well as inclusively for the combined data are shown in figure 1(left) as a function of $\left\langle N_{\text {part }}\right\rangle$. Data are independent on centrality, what means they scale with $\left\langle N_{\text {coll }}\right\rangle$, and consistent with QCD NLO predictions obtained
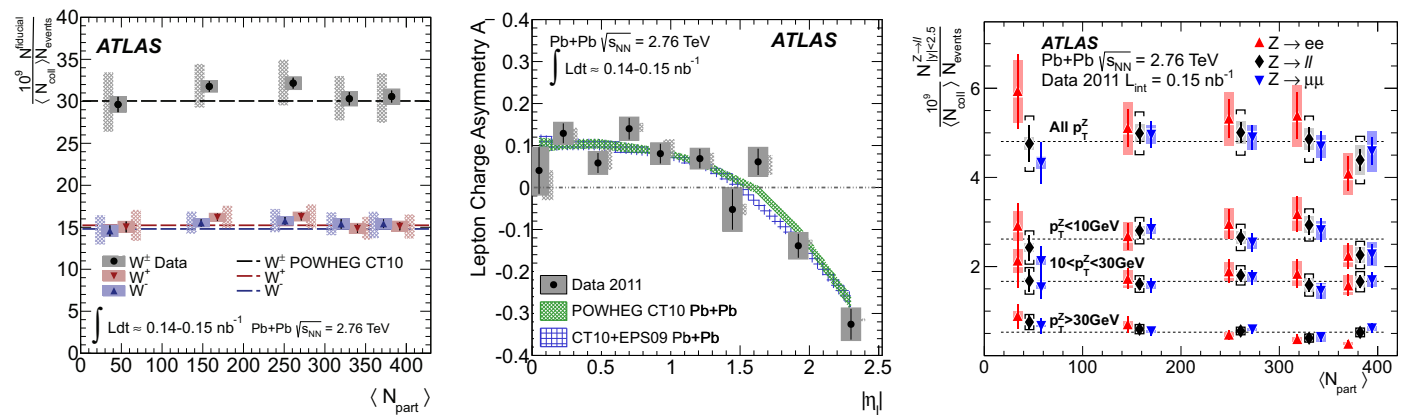

Figure 1. (left) $W$ boson production yields per binary collision as a function of $\left\langle N_{\text {part }}\right\rangle$ for $W^{+}, W^{-}$and $W^{ \pm}$bosons for combined muon and electron decay channels in the fiducial region defined in the text [11]. (middle) The lepton charge asymmetry $A_{\ell}$ from $W^{ \pm}$bosons as a function of absolute pseudorapidity measured in the fiducial region defined in the text [11]. (right) Centrality dependence of $Z$ boson yields divided by $\left\langle N_{\text {coll }}\right\rangle$. Results for $e e$ and $\mu \mu$ decay channels are shifted left and right, respectively, from their weighted average [12]. 
from POWHEG [15] MC with CT10 PDF [16]. In figure 1(middle) the lepton charge asymmetry defined in terms of differential production yields as:

$$
A_{\ell}\left(\eta_{\ell}\right)=\frac{\mathrm{d} N_{W^{+} \rightarrow \ell^{+} v_{\ell}} / \mathrm{d} \eta_{\ell}-\mathrm{d} N_{W^{-} \rightarrow \ell^{-} \bar{v}_{\ell}} / \mathrm{d} \eta_{\ell}}{\mathrm{d} N_{W^{+} \rightarrow \ell^{+} v_{\ell}} / \mathrm{d} \eta_{\ell}+\mathrm{d} N_{W^{-} \rightarrow \ell^{-} \bar{v}_{\ell}} / \mathrm{d} \eta_{\ell}}
$$

is shown as a function of $\left|\eta_{\ell}\right|$ and compared to NLO predictions with the CT10 PDF set, both with and without EPS09 [10] nuclear modifications. Both predictions are in agreement with data, so at the current precision this measurement is insensitive to nuclear modifications to the PDF.

Per event yields of $Z$ boson production in both electron and muon decay channels in $\mathrm{Pb}+\mathrm{Pb}$ collisions are shown in figure 1(right) as a function of $\left\langle N_{\text {part }}\right\rangle$ in bins of $p_{\mathrm{T}}^{Z}$. The results in both channels are consistent with each other and the fits using a constant (shown as dashed lines) confirm that the $Z$ boson per event yield obeys binary collision scaling.

ATLAS has also measured $W$ boson production in $p+\mathrm{Pb}$ collisions in the muon channel in a similar fiducial region as in the case of $\mathrm{Pb}+\mathrm{Pb}$ collisions [13]. The yields per $\mathrm{MB}$ events scaled by $\left\langle N_{\text {coll }}\right\rangle$ are shown separately for $W^{+}$and $W^{-}$in figure 2 for central (left) and peripheral (middle) events as a function of muon pseudorapidity $\eta_{\text {lab }}^{\mu}$. Data are compared to POWHEG calculations with CT10 PDF. In peripheral collisions the model calculations are lower than data for all values of $\eta_{\text {lab }}^{\mu}$. This may be due to the difficulty in definitively determining $\left\langle N_{\text {coll }}\right\rangle$ for a given centrality bin; in addition to the $\left\langle N_{\text {coll }}\right\rangle$ uncertainties shown in the figure, the normalization of each distribution depends on the choice of centrality model (Glauber or Glauber-Gribov Colour Fluctuation). There appears to be a dependence of the shape of the pseudorapidity distributions of both positively and negatively charged muons from $W$ bosons on centrality. The middle panels, displaying the ratios of the measured muon pseudorapidity distribution coming from $W^{ \pm}$bosons to that calculated with the model, suggest the presence of a slope in the most central collisions, which does not appear in peripheral collisions. The
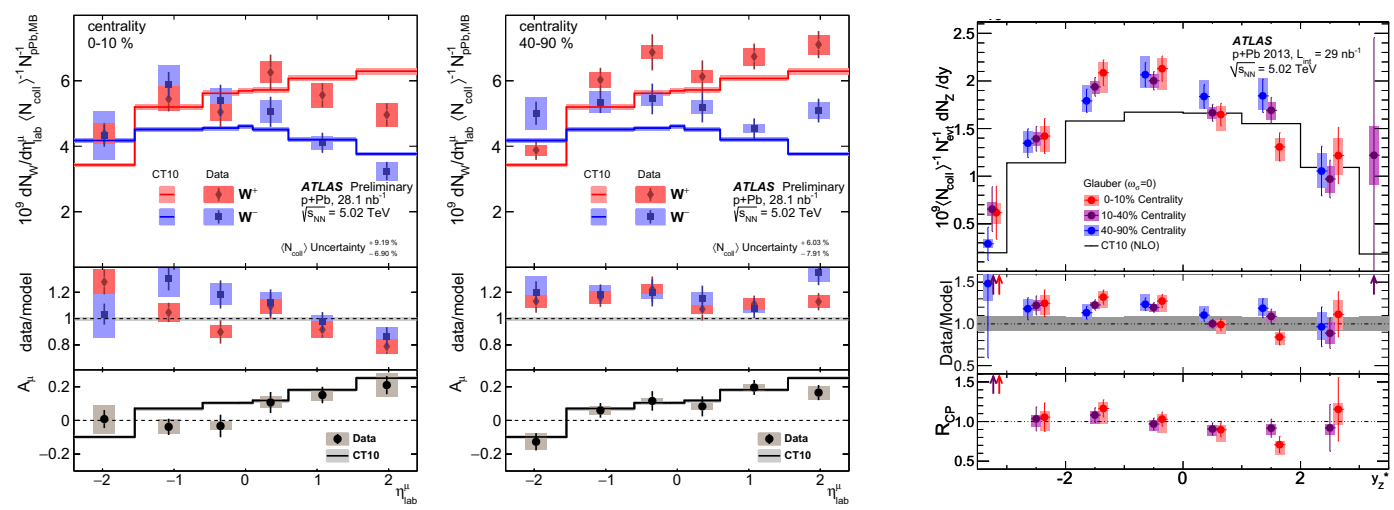

Figure 2. (left and middle) Production yields of $W \rightarrow \mu v$ in $p+\mathrm{Pb}$ collisions, scaled by $\left\langle N_{\text {coll }}\right\rangle$ and per $\mathrm{MB}$ event are shown as a function of the muon pseudorapidity in the laboratory frame for the centralities indicated in the plots, separately for $W^{+}$and $W^{-}$. The lower panels show the asymmetry defined in the text compared to the same model [13]. (right) Production yields of $Z \rightarrow \ell \ell,(\ell=e, \mu)$, in $p+\mathrm{Pb}$ collisions, scaled by $\left\langle N_{\text {coll }}\right\rangle$ and per $\mathrm{MB}$ event are shown as a function of the $Z$ boson rapidity in the centre-of-mass frame for the centralities indicated in the plot. The lower panel shows the ratio of yields in a given centrality to the yield in most peripheral collisions [14]. In all plots: Results are compared to the POWHEG model using CT10 PDFs and the ratio of neutrons and protons in $\mathrm{Pb}$ nuclei. The data-to-model ratios are shown in the middle panels. The error bars correspond to the statistical uncertainties, and error boxes correspond to the total systematic uncertainties. 


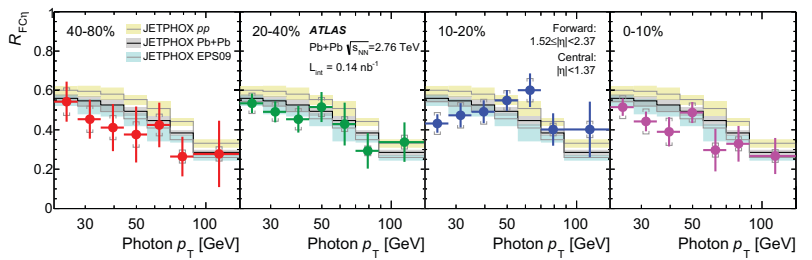

Figure 3. Ratios of yields of prompt photons as a function of $p_{\mathrm{T}}$ in forward $(1.52<|\eta|<$ 2.37) to central $(|\eta|<1.37)$ pseudorapidity regions measured in four centrality intervals [17]. Data are compared to MC predictions explained in text. Statistical and systematic uncertainties are shown as the bars and braces.

asymmetries shown in the lower panel of figure 2(left, middle) agree between the data and model, except in the Pb-going direction $\left(\eta_{\mathrm{lab}}^{\mu}<0\right)$ in the most central collisions.

For completeness ATLAS has investigated production of $Z$ bosons in $p+\mathrm{Pb}$ collisions [14]. It is worth noticing here that the asymmetric energy of the beams in $p+\mathrm{Pb}$ collisions results in a shift of the center-of-mass by 0.465 units of rapidity relative to the laboratory frame in the direction of the proton beam. $Z$ bosons were reconstructed in both electron and muon channels. In figure 2(right) dependence of the $Z$ boson yields in different centrality bins is shown as a function of the $Z$ boson rapidity in the centre-of-mass system $y_{Z}^{\star}$. The ratio of central to peripheral data, $R_{\mathrm{CP}}$, shown in the figure, is used to observe changes in rapidity distribution for different centrality bins in a model-independent way. Events with $40-90 \%$ centrality define the peripheral event selection, and two central selections, $0-10 \%$ and $10-40 \%$, are compared with it. It is observed that the asymmetry in $y_{Z}^{\star}$ is slightly larger in more central events. Integrated over $y_{Z}^{\star}$, the centrality dependence appears to be consistent with binary scaling.

ATLAS has also studied production of prompt photons produced in $\mathrm{Pb}+\mathrm{Pb}$ collisions [17]. Prompt photons have two primary sources: direct emission via quark-gluon Compton scattering or quarkantiquark annihilation, or in the fragmentation process through quark radiation of hard photons. To suppress the large background of non-prompt photons originating from the decays of neutral mesons in jets as well as photons from the fragmentation process, an isolation criterion has been applied. ATLAS has measured yields of prompt photons as a function of $p_{\mathrm{T}}$ in several centrality intervals in forward (F: $1.52<|\eta|<2.37)$ and central $(\mathrm{C}:|\eta|<1.37)$ pseudorapidity regions. In figure 3 the ratios of these yields are shown and compared to JETPHOX [18] predictions without and with nuclear modifications [10]. The precision of the measurement is not sufficient to indicate presence of nuclear modifications, however a slight preference of calculations incorporating isospin effects is observed.

ATLAS has performed the measurement of prompt and non-prompt $J / \Psi$ production in $p+\mathrm{Pb}$ collisions [19]. The $J / \Psi$ mesons were reconstructed using the dimuon decay mode in the fiducial phase space: $8<p_{\mathrm{T}}<30 \mathrm{GeV}$ and $\left|y^{\star}\right|<1$.94. A non-prompt $J / \Psi$ contribution, i.e. $J / \Psi$ from decay chains of $b$-quarks, was separated from prompt $J / \Psi$ by measuring displaced decay vertices. The measured forward $\left(y^{\star}>0\right)$ to backward $\left(y^{\star}<0\right)$ production ratio $R_{\mathrm{FB}}$ is shown in figure 4 as a function of $J / \Psi$ transverse momentum and the centre-of-mass rapidity. The measured distributions
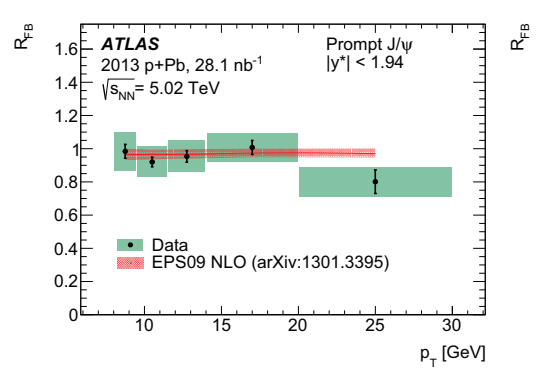

Figure 4. Forward-backward production ratio, $R_{\mathrm{FB}}$, for prompt $J / \Psi$ as a function of $p_{\mathrm{T}}$ (left) and $\left|y^{\star}\right|$ (right) [19]. Error bars show the statistical uncertainties and the shaded boxes show total uncertainties. EPS09 predictions are shown as indicated on the plots. 
are consistent with unity and no significant dependence on $p_{\mathrm{T}}$ or $y^{\star}$ is found. Data are also in good agreement with calculations including nuclear modifications (EPS09) [10] in LO and NLO.

\section{Charged-particle spectra and jets}

Experimental studies of charged-particles and jet production in $\mathrm{HI}$ collisions can directly reveal the properties of the QGP created in the collisions. One of the predicted consequences of QGP formation is 'jet quenching' that refers to the modification of parton showers initiated by hard-scattering processes which take place in QGP. It is also expected that the resulting spectrum of hadrons, which originate from parton fragmentation, is also modified.

ATLAS has measured the nuclear modification factor in $\mathrm{Pb}+\mathrm{Pb}, p+\mathrm{Pb}$ and $p p$ collisions both for charged particle spectra $[8,20,21]$ and for jet yields $[24,25]$.

The nuclear modification factor for yields of charged-particles produced in $\mathrm{Pb}+\mathrm{Pb}$ collisions has been studied by ATLAS in [21]. In the left panel of figure 5(upper) distributions of $R_{\mathrm{AA}}$ are shown for pseudorapidity range $|\eta|<2$ and five centrality intervals in the $p_{\mathrm{T}}$ range $0.5-150 \mathrm{GeV}$. The $R_{\mathrm{AA}}$ measured as a function of $p_{\mathrm{T}}$ shows a characteristic non-flat $p_{\mathrm{T}}$ shape which becomes more pronounced for the more central collisions. They first increase with increasing $p_{\mathrm{T}}$ reaching a maximum at $p_{\mathrm{T}} \approx 2 \mathrm{GeV}$, a feature commonly associated with the Cronin effect [22]. Then, $R_{\mathrm{AA}}$ decreases with higher $p_{\mathrm{T}}$ reaching a minimum at $p_{\mathrm{T}} \approx 7 \mathrm{GeV}$, where the charged-particle suppression is strongest. The suppression is strongest in the most central $0-5 \%$ collisions, although it is still noticeable even in the $60-80 \%$ centrality interval. Above this $p_{\mathrm{T}}, R_{\mathrm{AA}}$ generally increases with increasing $p_{\mathrm{T}}$ up to approximately $60 \mathrm{GeV}$ and then reaches a plateau. In the left panel of figure 5(lower), $R_{\mathrm{AA}}$ is shown as a function of the mean number of participating nucleons, $\left\langle N_{\text {part }}\right\rangle$, in the four characteristic transverse momentum intervals from the previous plot. In all these $p_{\mathrm{T}}$ intervals $R_{\mathrm{AA}}$ decreases with $N_{\text {part }}$,
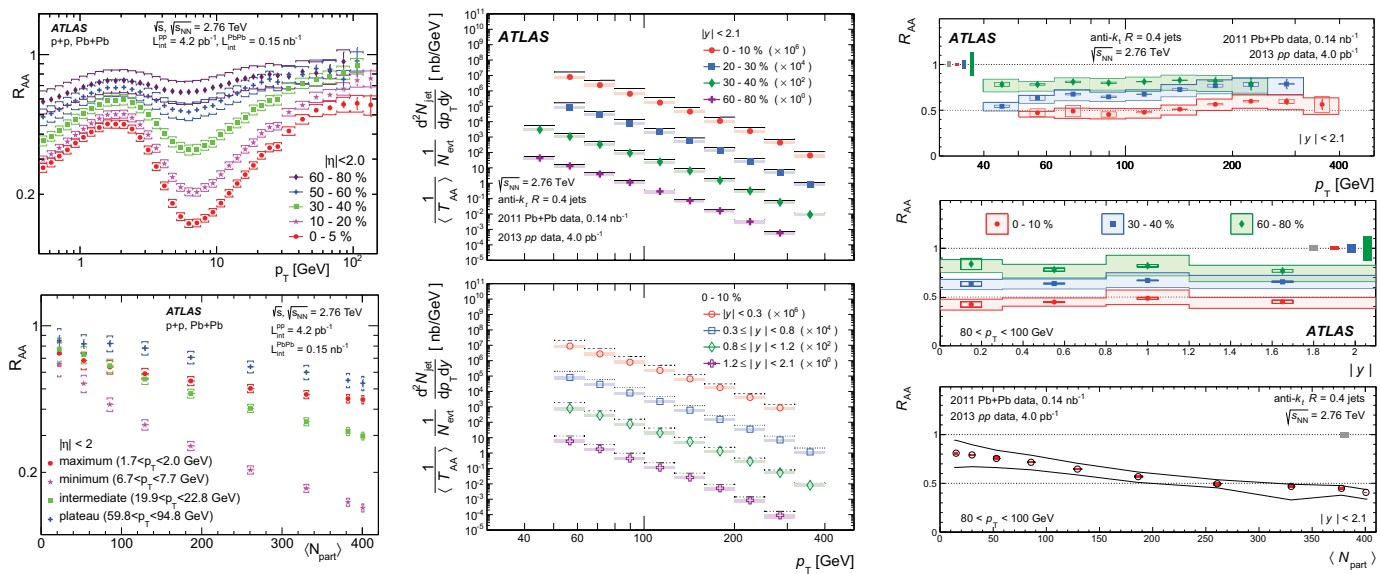

Figure 5. Left panel: The charged-particle $R_{\mathrm{AA}}$ as a function of $p_{\mathrm{T}}$ measured in several centrality intervals (upper) and as a function of $\left\langle N_{\text {part }}\right\rangle$ measured in four transverse momentum intervals (lower) [21]. Middle panel: The per-event jet yield in $\mathrm{Pb}+\mathrm{Pb}$ collisions, multiplied by $1 /\left\langle T_{\mathrm{AA}}\right\rangle$, as a function of $p_{\mathrm{T}}$. The upper plot shows the $|y|<2.1$ rapidity range in different centrality intervals. The lower plot shows the $0-10 \%$ centrality interval in different rapidity ranges. The solid and dashed lines represent the $p p$ jet cross section for the same rapidity interval scaled by the same factor. Right panel: Jet $R_{\mathrm{AA}}$ as a function of $p_{\mathrm{T}}$ for $|y|<2.1$ (upper), as a function of $|y|$ for $80<p_{\mathrm{T}}<100 \mathrm{GeV}$ (middle) in different centrality bins and as a function of $\left\langle N_{\text {part }}\right\rangle[24]$. 

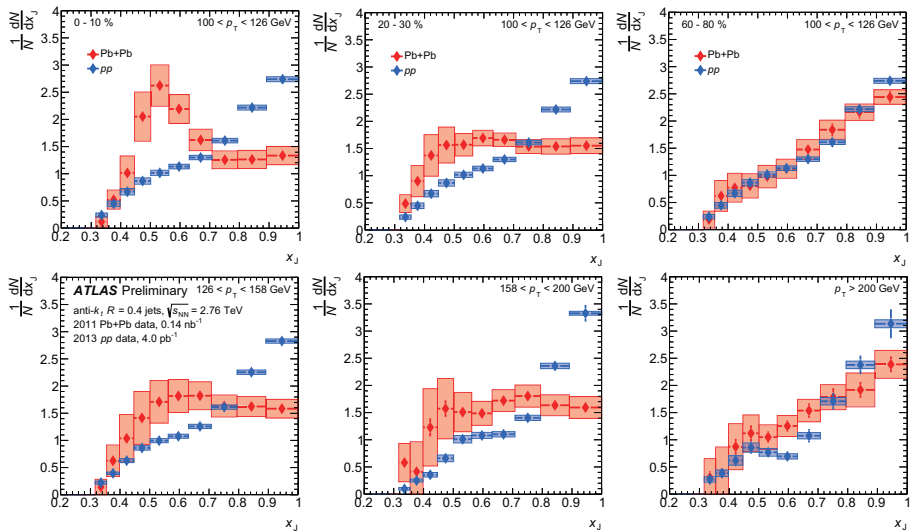

Figure 6. (upper) The $x_{\mathrm{J}}$ distributions for pairs with $100<p_{\mathrm{T}, 1}<$ $126 \mathrm{GeV}$ for different collision centralities. (lower) The $x_{\mathrm{J}}$ distributions for $0-10 \%$ centrality bin and for different ranges of the transverse momenta (indicated in the plots) of the leading jet. The $\mathrm{Pb}+\mathrm{Pb}$ data are shown in red, while the $p p$ distribution is shown for comparison in blue. Statistical uncertainties are indicated by the error bars while systematic uncertainties are shown with shaded boxes [26].

however the decrease is strongest for the $p_{\mathrm{T}}$ interval corresponding to the minimum and weakest in the plateau region. In the $p_{\mathrm{T}}$ ranges where the maximum and intermediate values of $R_{\mathrm{AA}}$ are measured the $R_{\mathrm{AA}}$ values are consistent for $N_{\text {part }}<100$, but then deviate at larger $N_{\text {part }}$. In the most central collisions, $R_{\mathrm{AA}}$ in the maximum interval is comparable to that in the plateau region.

Nuclear modification factors for jets production in $\mathrm{Pb}+\mathrm{Pb}$ collisions have been studied by the ATLAS experiment in [24]. In this analysis and also in the following ones discussed below, to reconstruct jets ATLAS uses anti- $k_{t}$ algorithm [23] with the radius parameter $R=0.4$.

The differential per-event jet yield in $\mathrm{Pb}+\mathrm{Pb}$ collisions, multiplied by $1 /\left\langle T_{\mathrm{AA}}\right\rangle$ is shown in figure 5(middle panel), in selected rapidity and centrality bins. The dashed lines represent the $p p$ jet cross sections for that same rapidity bin; the jet suppression is evidenced by the fact that the jet yields fall below these lines. In the right panel of figure 5, the jet $R_{\mathrm{AA}}$ is shown as a function of $p_{\mathrm{T}},|y|$ for different ranges in collision centrality and as a function of $\left\langle N_{\text {part }}\right\rangle$ for $80<p_{\mathrm{T}}<100 \mathrm{GeV}$. The $R_{\mathrm{AA}}$ is observed to increase weakly with $p_{\mathrm{T}}$. The $R_{\mathrm{AA}}$ shows no significant rapidity dependence. The $R_{\mathrm{AA}}$ decreases smoothly from the most peripheral collisions (smallest $\left\langle N_{\text {part }}\right\rangle$ ) to central collisions, where it reaches a minimal value of approximately 0.4 in the most central $1 \%$ of collisions.

Quarks and gluons in the shower passing through QGP may be scattered elastically or inelastically resulting in both deflection and energy loss of the constituents of the shower. Therefore a complete characterization of the effects of jet quenching requires measurements of both the single jet suppression and of jet fragmentation distributions. ATLAS has recently studied the transverse momentum balance in dijet pairs [26]. The momentum balance of the dijet system is expressed by the variable $x_{\mathrm{J}} \equiv p_{\mathrm{T}, 2} / p_{\mathrm{T}, 1}$, where $p_{\mathrm{T}, 1}$ and $p_{\mathrm{T}, 2}$ are the transverse momenta of the jets with the highest and second highest $p_{\mathrm{T}}$ in the event with $p_{\mathrm{T}}>25 \mathrm{GeV}$ and $|\eta|<2.1$. The pair is also required to have $\Delta \phi>7 \pi / 8$. The unfolded $x_{\mathrm{J}}$ distributions are shown in figure 6(upper), for pairs with $100<p_{\mathrm{T}, 1}<126 \mathrm{GeV}$ for different centrality intervals. The distribution in $p p$ collisions is shown on each panel for comparison. In the $60-80 \%$ centrality bin, where the effects of quenching are expected to be the smallest, the $\mathrm{Pb}+\mathrm{Pb}$ data are generally consistent with the $p p$ data. In more central $\mathrm{Pb}+\mathrm{Pb}$ collisions, the distributions become significantly broader than that in $p p$ collisions and the peak at $x_{\mathrm{J}} \sim 1$, corresponding to nearly-symmetric dijet events, is reduced. The distribution becomes almost flat over the range $0.5 \lesssim x_{\mathrm{J}} \lesssim 1$, before developing a peak at $x_{\mathrm{J}} \sim 0.5$ in the most central collisions. At larger values of $p_{\mathrm{T}, 1}$ the $x_{\mathrm{J}}$ distributions, shown in figure 6(lower), are observed to narrow, indicating that higher $p_{\mathrm{T}}$ dijets tend to be better balanced in momentum. This is consistent with a picture in which the fractional energy loss decreases with jet $p_{\mathrm{T}}$. The modifications observed in the $\mathrm{Pb}+\mathrm{Pb}$ data lessen with increasing $p_{\mathrm{T}, 1}$ and for pairs with $p_{\mathrm{T}, 1}>200 \mathrm{GeV}$ the maximum at $x_{\mathrm{J}} \sim 1$ is restored. 

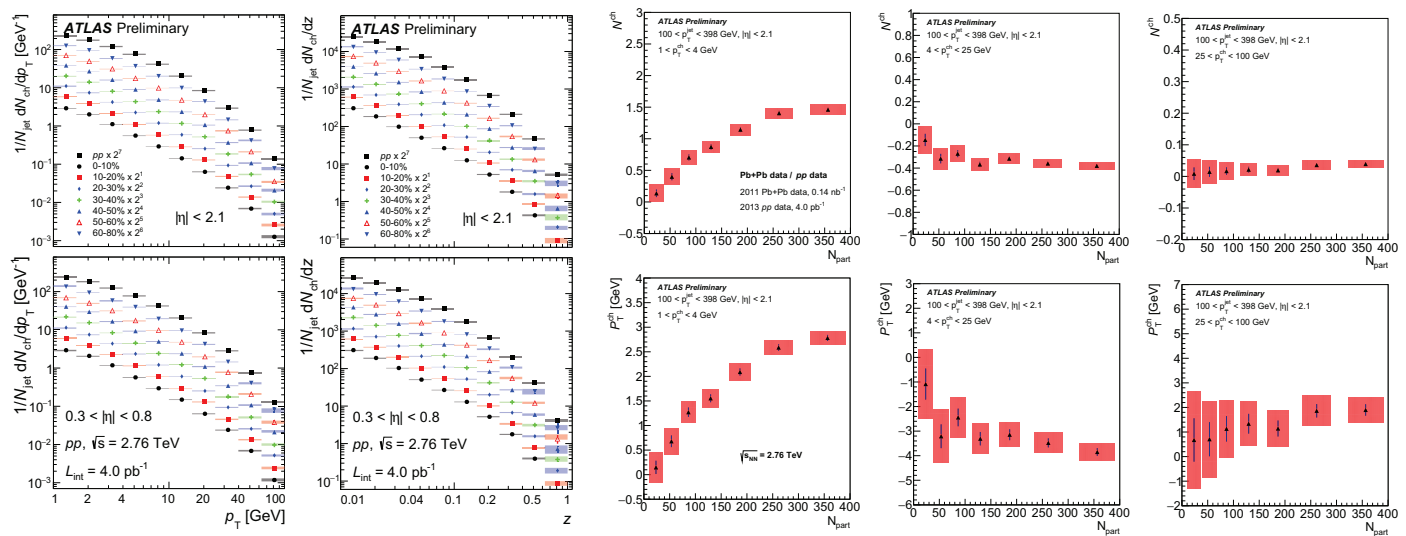

Figure 7. Left panel: Unfolded charged particle transverse momentum (left) and longitudinal momentum fraction (right) distributions, measured in $p p$ collisions and for seven centrality bins measured in $\mathrm{Pb}+\mathrm{Pb}$ collisions. Right panel: (upper) difference in the total yield of particles in a given $p_{\mathrm{T}}$ interval (indicated in the legend) measured in $\mathrm{Pb}+\mathrm{Pb}$ and the total yield of particles in the same $p_{\mathrm{T}}$ interval measured in $p p$ collisions, $N^{\mathrm{ch}}$; (lower): difference in the total transverse momentum of particles in a given $p_{\mathrm{T}}$ interval measured in $\mathrm{Pb}+\mathrm{Pb}$ and the total transverse momentum of particles measured in $p p, P_{\mathrm{T}}^{\mathrm{ch}}$. The differences are evaluated as a function of number of participating nucleons. In all plots: the error bars on the data points indicate statistical uncertainties while the shaded bands indicate systematic uncertainties [27].

Internal structure of jets in $\mathrm{Pb}+\mathrm{Pb}$ and $p p$ collisions ATLAS has studied in terms of longitudinal momentum fraction and transverse momenta of charged particles inside the jet [27]. Jet fragments having minimum $p_{\mathrm{T}}$ of $1 \mathrm{GeV}$ were measured within an angular range $\Delta R=0.4$ from the jet axis. The distributions of longitudinal momentum fraction and transverse momenta of charged particles inside jets with $p_{\mathrm{T}}$ in the interval $100-398 \mathrm{GeV}$ and in two overlapping pseudorapidity ranges are shown in figure 7(left panel) for $p p$ collisions and several centrality intervals for $\mathrm{Pb}+\mathrm{Pb}$ collisions. The distributions exhibit a difference in shape between central HI collisions and peripheral HI collisions or $p p$ reference. To better quantify the observed trends, the differences between integrals of distributions of charged particles transverse momenta measured in HI collisions and in $p p$ collisions have been calculated and are shown in the right panel of figure 7(upper). The differences are shown for three ranges of charged particles transverse momenta (indicated in the plots) as a function of centrality expressed in terms of the number of participants. There is clear, almost logarithmic, increase of yields with centrality observed for particles with $1<p_{\mathrm{T}}<4 \mathrm{GeV}$, a depletion is seen for particles with $4<p_{\mathrm{T}}<25 \mathrm{GeV}$, and a slight enhancement is seen for particles with $25<p_{\mathrm{T}}<100 \mathrm{GeV}$. Similarly, differences of the total transverse momentum of charged particles in $\mathrm{HI}$ and $p p$ collisions have been calculated and are shown in figure 7(lower). The changes in the total transverse momentum follow the trends seen in the yields. The significance of the increase of the total transverse momentum in the high- $p_{\mathrm{T}}^{\mathrm{ch}}$ region is higher than the significance of the increase of yields of charged particles seen in the same region.

To help to disentangle the contributions to the observed dijet asymmetries from the unequal path lengths of the showers in the medium from fluctuations in the energy loss process itself, ATLAS measured the correlations between jets that are produced at small relative angles [28]. Neighbouring jet pairs include jets originating from the same hard interaction, but also jets from different hard interactions. The latter are not of interest in this analysis, and are subtracted statistically. The remaining neighbouring jet pairs result primarily from hard radiation by the parton that occurs early 


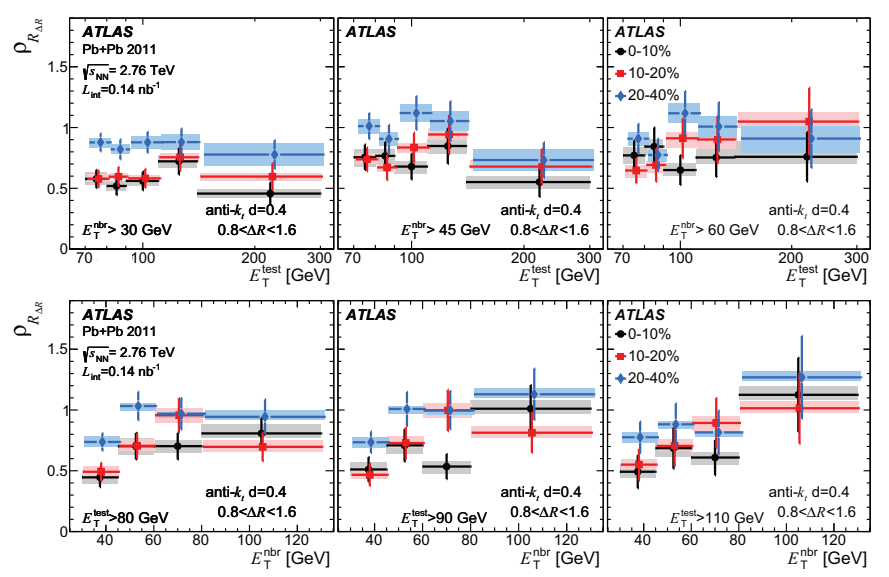

Figure 8. The ratio of $R_{\Delta R}$ for three bins of collision centrality to those in $40-80 \%$ collisions [28]. The ratio $\rho_{R_{\Delta R}}$ is evaluated as a function of $E_{\mathrm{T}}^{\text {test }}$ for three different choices of lower bound on $E_{\mathrm{T}}^{\mathrm{nbr}}$ (upper) and as a function of $E_{\mathrm{T}}^{\mathrm{nbr}}$ for three different choices of lower bound on $E_{\mathrm{T}}^{\text {test }}$ (lower). The error bars represent statistical uncertainties and the shaded bands the systematic uncertainties. The data points are shifted along the horizontal axis for clarity.

in the process of the shower formation. Generally, two neighbouring jets originating from the same hard scattering should have more similar path lengths in the medium compared to the two jets in the dijet measurement. Therefore measuring neighbouring jets could probe differences in their quenching that do not result primarily from difference in path length. More generally, measurements of the correlated production of jets in the same parton shower may provide more detailed insight into the modification of the parton shower in the QGP beyond the subsequent quenching of the resulting jets. The rate of neighbouring jets, $R_{\Delta R}$, that accompany 'test' jet within a given range of angular distance $\Delta R=\sqrt{(\Delta \phi)^{2}+(\Delta \eta)^{2}}$ is measured in bins of transverse energies of test jets, $E_{\mathrm{T}}^{\text {test }}$, and neighbouring jets, $E_{\mathrm{T}}^{\mathrm{nbr}}$, in several centrality intervals. In figure 8 the ratios of $R_{\Delta R}$ for three bins of collision centrality to those in $40-80 \%$ collisions is shown as a function of both $E_{\mathrm{T}}^{\text {test }}$ and $E_{\mathrm{T}}^{\text {nbr }}$, in each case for different lower bounds of the other variable. The $\rho_{R_{\Delta R}}$ distributions do not exhibit any strong dependence on $E_{\mathrm{T}}^{\text {test }}$. The suppression factor $\rho_{R_{\Delta R}}$ of the most central collisions is at the level of $0.5-0.7$ for all three lower bounds on $E_{\mathrm{T}}^{\mathrm{nbr}}$. The suppression seems to become less pronounced with decreasing centrality. This is qualitatively consistent with the observation of the centrality-dependent suppression of inclusive jet yields. Contrary to a modest dependence of $\rho_{R_{\Delta R}}$ on the test-jet $E_{\mathrm{T}}$, the $\rho_{R_{\Delta R}}$ evaluated as a function of $E_{\mathrm{T}}^{\mathrm{nbr}}$ suggests a decrease of suppression with increasing $E_{\mathrm{T}}^{\mathrm{nbr}}$. Such a decrease in suppression with increasing $E_{\mathrm{T}}^{\mathrm{nbr}}$ may in fact be expected. The jet quenching is generally expected to depend on the initial parton energy, but if the splitting happens such that the two partons have similar energy, their quenching would likely be comparable due to similar in-medium path-length traveled by the two partons forming neighbouring jets. Thus, in the configuration of $E_{\mathrm{T}}^{\text {test }} \approx E_{\mathrm{T}}^{\mathrm{nbr}}$ the per-test-jet normalization effectively removes the impact of the suppression.

\section{Bulk collectivity}

The two-particle correlation function in pseudorapidity $C_{\mathrm{N}}\left(\eta_{1}, \eta_{2}\right)$ has been measured by the ATLAS experiment in $\mathrm{Pb}+\mathrm{Pb}, p+\mathrm{Pb}$ and $p p$ collisions [30]. In order that it contains only dynamical fluctuations, the correlation function has been corrected for any residual multiplicity dependence in the average shape $\langle N(\eta)\rangle$. In order to quantify the features of the correlation function, it is essential to estimate and separate the contributions from the short-range correlations (SRC) and the long-range correlations (LRC). The SRC reflect correlations within the same source, while the LRC reflects FB-asymmetry of the number of sources. A method based on different behaviour of the correlation function for SRC and LRC for the same-charge and opposite-charge particles [30], was developed and used to subtract the SRC from the total correlation function. The correlation function is measured 

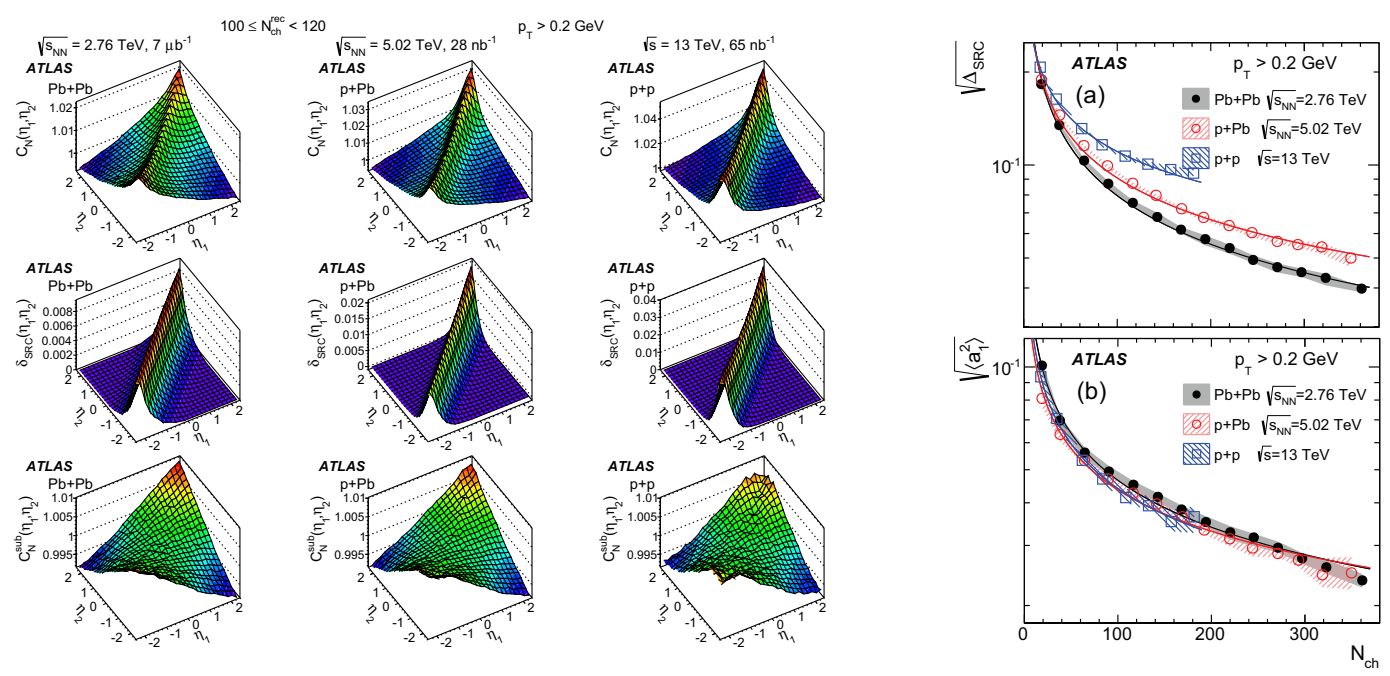

Figure 9. Left panel: The distributions of correlation functions $C_{\mathrm{N}}\left(\eta_{1}, \eta_{2}\right)$ (top row), the estimated short-range component $\delta_{\mathrm{SRC}}\left(\eta_{1}, \eta_{2}\right)$ (middle row) and long-range component $C_{\mathrm{N}}^{\text {sub }}$ (bottom row). They are shown for events with $100 \leq N_{\mathrm{ch}}^{\mathrm{rec}}<120$ in $\mathrm{Pb}+\mathrm{Pb}, p+\mathrm{Pb}$ and $p p$ collisions. Right panel: (a) The estimated magnitude of the short-range component $\sqrt{\Delta_{\mathrm{SRC}}}$ and (b) the coefficient $\sqrt{\left\langle a_{1}^{2}\right\rangle}$ as a function of $N_{\text {ch }}$ for all-charge pairs in $\mathrm{Pb}+\mathrm{Pb}$, $p+\mathrm{Pb}$ and $p p$ collisions. The shaded bands represent systematic uncertainties, and the statistical uncertainties are smaller than the symbols [30].

using charged particles in the pseudorapidity range $|\eta|<2.4$ with transverse momentum $p_{\mathrm{T}}>0.2$ $\mathrm{GeV}$, and it is measured as a function of event multiplicity, defined by a number of charged particles with $|\eta|<2.5$ and $p_{\mathrm{T}}>0.4 \mathrm{GeV}$. The measured $C_{\mathrm{N}}\left(\eta_{1}, \eta_{2}\right)$ is shown in the upper row of the left panel of figure 9 for different collision systems and for the multiplicity range $100<N_{\mathrm{ch}}^{\mathrm{rec}}<120$. The resulting SRC denoted as $\delta_{\mathrm{SRC}}\left(\eta_{1}, \eta_{2}\right)$ and LRC estimate denoted as $C_{\mathrm{N}}^{\text {sub }}\left(\eta_{1}, \eta_{2}\right)$ are shown in the middle and bottom rows, respectively. The magnitude of the $\mathrm{SRC}$ in $p+\mathrm{Pb}$ is observed to be larger in the proton-going direction than in the lead-going direction, reflecting the fact that the particle multiplicity is smaller in the proton-going direction. However, this forward-backward asymmetry in $p+\mathrm{Pb}$ collisions is mainly associated with the SRC component, and the $C_{\mathrm{N}}^{\mathrm{sub}}\left(\eta_{1}, \eta_{2}\right)$ distribution shows very little asymmetry. $C_{\mathrm{N}}\left(\eta_{1}, \eta_{2}\right)$ distributions show significant differences between the three systems, which is mainly due to their differences in $\delta_{\mathrm{SRC}}\left(\eta_{1}, \eta_{2}\right)$. In fact the estimated long-range component $C_{\mathrm{N}}^{\mathrm{sub}}\left(\eta_{1}, \eta_{2}\right)$ shows similar shape and similar overall magnitude for the three systems.

The magnitude of the SRC is quantified by $\delta_{\mathrm{SRC}}\left(\eta_{1}, \eta_{2}\right)$ averaged over two-particle pseudorapidity phase space $\Delta_{\mathrm{SRC}}=\left(1 / 4 Y^{2}\right) \int \delta_{\mathrm{SRC}}\left(\eta_{1}, \eta_{2}\right) \mathrm{d} \eta_{1} \mathrm{~d} \eta_{2}$, where $Y=2.4$. The corresponding contribution of the SRC at the single-particle level is $\sqrt{\Delta_{\text {SRC }}}$ and is shown in the right panel of figure 9(upper) as a function of $N_{\mathrm{ch}}$ for different charge combinations in the three collision systems. The strength of the SRC always decreases with $N_{\mathrm{ch}}$ and is larger for smaller collision systems.

To quantify the LRC the correlation function $C_{\mathrm{N}}^{\text {sub }}\left(\eta_{1}, \eta_{2}\right)$ has been decomposed into orthogonal Legendre polynomials. From the fit to the measured distribution only the coefficient $\left\langle a_{1}^{2}\right\rangle$ is significantly different from zero. Distributions of $\sqrt{\left\langle a_{1}^{2}\right\rangle}$ as a function of $N_{\mathrm{ch}}$ for different collision systems are shown in the right panel of figure 9(bottom). The LRC signal is found to be similar between the three collision systems, which is a non-trivial result as the distribution of $N_{\mathrm{ch}}$ as well as the sizes of the overlap region are very different between the three collision systems. 


\section{Ultra-peripheral collisions}

In the case of nuclei colliding at large impact parameters, the strong interaction is not active. However, due to their strong electromagnetic fields, the nuclei can coherently produce high energy nearly real photons. That means that in these so-called ultra-peripheral collisions (UPC) one can study twophoton processes, because relatively small couplings are compensated with very high photon-photon luminosity which scales with the nuclear electric charge as $Z^{4}$. The maximum energy for coherent photons emitted from relativistic nucleus is $\gamma \hbar c / R$, where $\gamma$ is the Lorentz factor of one nucleus and $R$ is the nuclear radius. This is about $75 \mathrm{GeV}$ for the $\mathrm{Pb}+\mathrm{Pb}$ collisions at $\sqrt{s_{\mathrm{NN}}}=5.02 \mathrm{TeV}$.

ATLAS has measured cross sections for exclusive dimuon production in UPC of lead nuclei for large dimuon invariant mass $M_{\mu \mu}>10 \mathrm{GeV}$ [31]. The cross sections were measured by selecting events using a single-muon trigger, in association with an otherwise low-multiplicity event. The events are required to have a primary vertex formed solely from two oppositely-charged muons. Events with QED final-state radiation (FSR) are subtracted by removing pairs with large acoplanarity (defined as $1-|\Delta \phi| / \pi)$. The events are then corrected for muon trigger and reconstruction efficiency, for vertex efficiency, and for the expected losses due to FSR. The results are compared directly with calculations from STARLIGHT 1.1 [32], as a function of the dimuon pair mass $\left(M_{\mu \mu}\right)$ and pair rapidity $\left(Y_{\mu \mu}\right)$ both defined in terms of the total four-momentum. The kinematic coverage is substantially larger than in any previous HI measurement, with pair rapidities out to $\left|Y_{\mu \mu}\right|<2.4$ and pair masses ranging from $10<M_{\mu \mu}<100 \mathrm{GeV}$.

The measured cross sections as a function of dimuon kinematic variables are shown in figure 10 . The mass distribution is shown for two (overlapping) rapidity regions $\left(\left|Y_{\mu \mu}\right|<2.4\right.$ and $\left.\left|Y_{\mu \mu}\right|>1.6\right)$ to show both the full distribution, and to focus on the forward region. The rapidity distribution is shown for three ranges of dimuon invariant mass $\left(10<M_{\mu \mu}<20 \mathrm{GeV}, 20<M_{\mu \mu}<40 \mathrm{GeV}, 40<M_{\mu \mu}<\right.$ $100 \mathrm{GeV}$ ). Error bars show statistical uncertainties while the grey bands show the combined systematic uncertainties, including the $7 \%$ global luminosity uncertainty. The MC statistical uncertainties, which are negligible in most cases, are not shown. STARLIGHT is in good agreement with the data over the full kinematic range, within the quoted uncertainties. This suggests that the nuclear electromagnetic fields are reasonably described by the equivalent photon approximation and that the nuclear form factor is adequately implemented in STARLIGHT.

Integrating the distributions in figure 10 gives the total cross section in the fiducial acceptance $\sigma\left(\mathrm{Pb}+\mathrm{Pb} \rightarrow \mu^{+}+\mu^{-}+\mathrm{Pb}^{(\star)}+\mathrm{Pb}^{(\star)}\right)=32.2 \pm 0.3$ (stat) ${ }_{-3.4}^{+4.0}$ (sys) $\mu$ b to be compared with $31.64 \pm 0.04 \mu b$ from the STARLIGHT prediction.
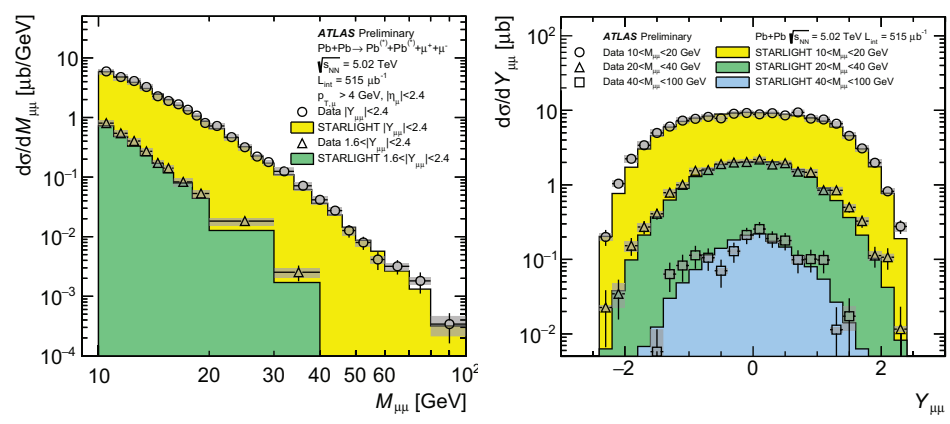

Figure 10. Cross section for dimuon production in UPC, as a function of pair mass (left) and pair rapidity (right) [31]. Data indicated by symbols are compared to STARLIGHT predictions shown by solid histograms. Error bars indicate statistical uncertainties while the gray band indicates the combined systematic uncertainties. 


\section{Summary}

Recent results from the ATLAS experiment related to the study of Quark-Gluon Plasma via both hard and soft probes have been briefly presented. For more details and in depth discussion of the measurements the reader is referred to the original publications. All ATLAS heavy-ion results are available from https://twiki.cern.ch/twiki/bin/view/AtlasPublic/HeavyIonsPublicResults This work was partly supported by the National Science Centre of Poland under grant number UMO2012/05/B/ST2/02480.

\section{References}

[1] STAR Collaboration, J. Adams, et al., Nucl. Phys. A 757, 102 (2005). [nucl-ex/0501009]

[2] BRAHMS Collaboration, I. Arsene, et al., Nucl. Phys. A 757, 1 (2005). [nucl-ex/0410020]

[3] PHOBOS Collaboration, B.B. Back, et al., Nucl. Phys. A 757, 28 (2005). [nucl-ex/0410022]

[4] PHENIX Collaboration, K. Adcox et al., Nucl. Phys. A 757, 184 (2005). [nucl-ex/0410003]

[5] N. Armesto and E. Scomparin, Eur. Phys. J. Plus 131, 52 (2016). [nucl-ex/1511.02151]

[6] ATLAS Collaboration, JINST 3, S08003 (2008). https://cds.cern.ch/record/1129811

[7] ATLAS Collaboration, Phys. Lett. B 707, 330-348 (2012). [nucl-ex/1108.6018]

[8] ATLAS Collaboration, Eur. Phys. J. C 76, 199 (2016). [hep-ex/1508.00848]

[9] V. Guzey, M. Strikman, Phys. Lett. B 633245 (2006). [hep-ph/0505088]

[10] K. Eskola et al., J. High Energy Phys. 04, 065 (2009). [hep-ph/0902.4154]

[11] ATLAS Collaboration, Eur. Phys. J. C 75 (2015) 23. [hep-ex/14084674]

[12] ATLAS Collaboration, Phys. Rev. Lett. 110, 022301 (2013). [hep-ex/1210.6486]

[13] ATLAS Collaboration, ATLAS-CONF-2015-056, http://cds.cern.ch/record/2055677

[14] ATLAS Collaboration, Phys. Rev. C 92, 044915 (2015). [hep-ex/1507.06232]

[15] S. Alioli, P. Nason, C. Oleari, E. Re, J. High Energy Phys. 07, 60 (2008). [hep-ph/0805.4802]

[16] H.-L. Lai et al., Phys. Rev. D 82, 074024 (2010). [hep-ph/1007.2241]

[17] ATLAS Collaboration, Phys. Rev. C 93, 034914 (2016). [hep-ex/1506.08552]

[18] P. Aurenche et al., Phys. Rev. D 73, 094007 (2006). [hep-ph/0602133]

[19] ATLAS Collaboration, Phys. Rev. C 92, 034904 (2015). [hep-ex/1505.08141]

[20] ATLAS Collaboration, submitted to Phys. Lett. B. [hep-ex/1605.06436]

[21] ATLAS Collaboration, J. High Energy Phys. 09, 50 (2015). [hep-ex/1504.04337]

[22] J.W. Cronin et al., Phys. Rev. D 11, 3105 (1975)

[23] M. Cacciari, G.P. Salam, G. Soyez, J. High Energy Phys. 04, 063 (2008). [hep-ph/0802.1189]

[24] ATLAS Collaboration, Phys. Rev. Lett. 114, 072302 (2015). [hep-ex/1411.2357]

[25] ATLAS Collaboration, Phys. Lett. B 748 (2015) 392. [hep-ex/1412.4092]

[26] ATLAS Collaboration, ATLAS-CONF-2015-052, http://cds.cern.ch/record/2055673

[27] ATLAS Collaboration, ATLAS-CONF-2015-055, http://cds.cern.ch/record/2055676

[28] ATLAS Collaboration, Phys. Lett. B 751, 376 (2015). [hep-ex/1506.08656]

[29] ATLAS Collaboration, submitted to Phys. Lett. B. [hep-ex/1605.06436]

[30] ATLAS Collaboration, submitted to Phys. Rev. C. [hep-ex/1606.08170]

[31] ATLAS Collaboration, ATLAS-CONF-2016-025, http://cds.cern.ch/record/2157689

[32] S. Klein and J. Nystrand, The STARLIGHT website, http://starlight.hepforge.org 